\title{
Introducing routine HIV screening for patients on an internal medicine residency inpatient service: a quality improvement project
}

\author{
Leslie J Padrnos, Patrick J Barr, Christine L Klassen, Heather E Fields, Natalya Azadeh, Neil Mendoza, Rayya A Saadiq, \\ Emanuel M Pauwels, Christopher S King, Andrew A Chung, Kenneth K Sakata, and Janis E Blair \\ Mayo Clinic Arizona, USA
}

\begin{abstract}
The US Centers for Disease Control and Prevention (CDC) recommend human immunodeficiency virus (HIV) screening for all persons aged 13 to 64 years who present to a health care provider. We sought to improve adherence to the CDC guidelines on the Internal Medicine Resident Hospital Service. We surveyed residents about the CDC guidelines, sent email reminders, provided education, and engaged them in friendly competition. Credit for guideline adherence was awarded if an offer of HIV screening was documented at admission, if a screening test was performed, or if a notation in the resident sign out sheet indicated why screening was not performed.
\end{abstract}

We examined HIV screening of a postintervention group of patients admitted between August 8, 2012, and June 30, 2013, and compared them to a preintervention group admitted between August 1, 2011, and June 30, 2012. Postintervention offers of HIV screening increased significantly $(7.9 \%$ [44/559] vs 55.5\% [300/541]; $\mathrm{P}<.001)$, as did documentation of residents' contemplation of screening $(8.9 \%$ [50/559] vs $67.5 \%$ [365/541]; $\mathrm{P}<.001)$. A significantly higher proportion of HIV screening tests was ordered postintervention $(7.7 \%$ [43/559] vs $44.4 \%$ [240/541]; $\mathrm{P}<.001)$. Monthly HIV screening documentation ranged from $0 \%(0 / 53)$ to $17 \%(9 / 53)$ preintervention, whereas it ranged from $30.6 \%$ $(11 / 36)$ to $100 \%(62 / 62)$ postintervention.

HIV screening adherence can be improved through resident education, friendly competition, and system reminders. Barriers to achieving sustained adherence to the CDC guidelines include a heterogeneous patient population and provider discomfort with the subject.

\section{Problem}

Quality and safety in the practice of medicine are emerging fields, and graduate medical education programs are increasingly aware of their importance in the educational process. The Accreditation Council for Graduate Medical Education (ACGME) Common Program Requirements of 2009 state that "Residents must demonstrate the ability to investigate and evaluate their care of patients... and to continuously improve patient care based on constant self-evaluation and life-long learning."[1] Residency programs implement this requirement in a variety of ways.

In 2006, the US Centers for Disease Control and Prevention (CDC) updated the recommendation regarding identification of HIVseropositive patients by advising all persons aged 13 to 64 years who present to a health care provider should be screened for HIV, irrespective of the presence of risk factors.

We conducted a quality improvement (QI) project to educate internal medicine residents on the 2006 CDC HIV-screening guidelines, with the goal of improving adherence to those guidelines for patients admitted to our internal medicine resident hospital service (IMResHospSvc). The QI project was conducted between August 8, 2012 and June 30, 2013, in our 268 bed, tertiary care hospital for adult patients in Phoenix, Arizona.

\section{Background}

The 1996 human immunodeficiency virus (HIV) screening recommendations from the US Centers for Disease Control and Prevention (CDC) advised that patients should be tested for HIV if risk factors for HIV infection are present (including, but not limited to, sexually transmitted diseases, men who have sex with men, use of injection drugs, exchange of sex for money, or a history of blood transfusion between 1978 and 1985).[2] However, because subsequent studies indicated that $7 \%$ to $51 \%$ of HIV-seropositive patients had no risk factors, [3][4] and that screening based entirely on reported risk factors resulted in missed diagnoses in $74 \%$ (79/107) of cases,[5] the subsequent 2006 CDC recommendations for identifying HIV-seropositive patients advised that all persons aged 13 to 64 years who present to a health care provider should be screened for HIV, irrespective of the presence of risk factors.[6]

The 2012 estimated prevalence of HIV and AIDS in Arizona was 233 per 100000 , with approximately $8.9 \%$ of patients with HIV and AIDS having no reportable risk factors.[7] In addition, the Arizona Department of Health Services has estimated that up to $25 \%$ of the women with new HIV infections and up to $11 \%$ of men with new HIV infections reported no HIV risk factors.[8]

\section{Baseline measurement}

Prior to designing the project, retrospective assessment of documentation regarding HIV screening was measured for the IMResHospSvc over the course of one month. The electronic medical record was reviewed for all admissions to the internal 


\section{BMJ Quality Improvement Reports}

medicine residency for one month. The proportion of patients admitted to the service between the ages of 13 to 64 years old with documentation of HIV screening offered or discussed was $9 \%$ $(\mathrm{N}=50 / 559)$.

See supplementary file: ds6270.docx - "PDSACycle and InterventionTable"

\section{Design}

The QI project was conducted between August 8, 2012 and June 30,2013 , in our 268-bed, tertiary care hospital for adult patients in Phoenix, Arizona

The IMResHospSvc was composed of two teams, each consisting of three interns (physicians in their first postgraduate year (PGY1)) and one senior (PGY2 or PGY3) resident. Interns on each team performed admissions under direct supervision of the senior resident and reported to an attending staff physician. One chief medical resident oversaw resident education each month.

This project was deemed exempt by the Mayo Clinic Institutional Review Board. The QI team identified potential barriers to implementation of HIV screening prior to initiation, including physician pushback, legal concerns, and patient pushback. In anticipation, the QI team provided information to the residents about 1) the 2006 CDC recommendations; 2) national and state legal issues regarding testing; and 3) screening test details. We also disseminated printed scripts and a flow chart to simplify the screening process and ensure appropriate handling of test results.

We faced several expected logistic barriers to implementation, including: 1) a rotating cohort of residents on the IMResHospSvc every month; 2) an influx of rotating residents from external institutions unfamiliar with the QI project; and 3) the advanced age (median age, 61 years) of patients admitted to our hospital. These were addressed with educational provisions, including a focus on HIV in the elderly.

Broadly, the interventions were either active or passive in nature. Active interventions included friendly competition, verbal education, and reminders, whereas passive interventions involved email, educational pamphlets, and workroom reminders.

Definitions

- "Patients who qualified for screening" were defined as adult patients hospitalized on the IMResHospSvc who were younger than 64 years old. Exclusion criteria included known HIV/AIDS diagnosis, inability to provide consent for screening, or prior HIV screening completed within the past year. As the project progressed, residents were encouraged to document whether or not HIV screening was "not indicated" in a particular patient based on clinical judgment such as a terminal diagnosis with limited life expectancy

- "HIV documentation" was defined as documentation of a discussion about HIV testing with the patient either in the electronic health record (EHR), the admission history and physical examination, or the discharge summary

- "HIV contemplation" was defined as any of the following: 1) HIV documentation; 2) HIV test ordered; or 3) IMResHospSvc sign-out sheet indicating patient acceptance or refusal of HIV screening test

We followed a plan, do, study, act (PDSA) paradigm to discuss, prioritize, and serially implement potential interventions. Each PDSA cycle correlated with a one month rotation cycle (tables 1 and 2). Interventions were modified monthly based on screening contemplation rates, resident feedback, and our perceptions of residents' accomplishments and failures. Formal surveys were sent to all rotating residents after their rotation, and feedback was reviewed in real time by the QI planning team.

\section{Methods of evaluation}

For each PDSA cycle, the QI team reviewed the HIV documentation in the admission history and physical examination, the discharge summary, and the sign out sheets for every patient admitted to the IMResHospSvc. The effectiveness of the intervention was determined by the proportion of hospital admissions with HIV screening contemplation during each cycle. We prospectively collected data on HIV documentation and screening tests for inpatients for the 11 month QI postintervention period (August 8, 2012 to June 30,2013 ) and compared it to patient data for the preintervention period prior to initiation of the project (August 1 , 2011 to June 30,2012 ).

\section{Strategy}

PDSA cycle 1: For our primary intervention, all residents on the IMResHospSvc service were presented with a one hour lecture delivered by a second year resident at morning report describing CDC recommendations, methods of available HIV testing, ordering process, potential approaches to patient questions, follow up reporting, and documentation of results. Residents were provided with a flow sheet describing steps to take based on test results, as well as H\&P templates including an HIV screening component. They were instructed to dictate "HIV screening" as a component of their admission plan, and include HIV screening status on the team sign-out sheet. The results of all HIV tests performed were reviewed by the QI team to ensure that follow-up was appropriate. At the completion of the first month, an anonymous electronic survey was sent to residents on the hospital services asking for input regarding individual's barriers to screening compliance.

PDSA cycle 2: The following changes were made following input from the first month cycle. The same one hour lecture was provided but this month it was delivered by the chief resident. Senior residents were contacted personally by the QI team to encourage and remind first year residents to screen patients, and the chief resident verbally reminded post-call first year residents to screen patients admitted overnight if it had not yet been done on a daily basis. Brief HIV facts were emailed to residents on service on a weekly basis as a reminder of the need for screening. At the conclusion of the month, the same electronic survey was sent, and chart review by the QI team was performed as described above for 
cycle 1.

PDSA cycle 3: By the third month of the intervention, all interns and senior residents on service had already seen the lecture on previous months; therefore it was not presented again. However, the chief resident continued to remind residents to screen patients admitted, weekly HIV facts were sent by email, and an email was sent at the beginning of the month to senior residents (including the night float) to remind first year residents to screen at admission.

PDSA cycle 4: In month four after institution of learning interventions given to medical residents regarding HIV screening guidelines, all active interventions were discontinued. No morning report was given regarding screening recommendations and the Chief Medical Residents no longer intervened to remind residents to screen patients within the recommended age range.

PDSA cycle 5: Again in this month no active interventions were performed. However, at this point the year, second year residents were introduced to the IMResHospSvc service as senior residents, all of whom are actively involved in the current QI project. There was one PGY-2 on each IMResHospSvc team. Additionally, a visiting senior resident was present on one of the two teaching services.

PDSA cycle 6: PGY-2 residents continued to act as senior residents on both IMResHospSvc services. No active interventions, such as morning report and reminder emails, were taken by the chiefs. In this cycle, visiting PGY-1 residents were introduced; one to each medical team, and no active intervention was requested for these visiting residents. It was left to the discretion of the IMResHospSvc senior or fellow PGY-1 residents to inform the visiting PGY-1 residents of the screening guidelines that are being followed. Of note, the visiting residents do come from an institution where almost all patients within the screening age are screened for HIV in the emergency department.

PDSA cycle 7: No active intervention was taken for continuing Mayo Clinic residents regarding HIV screening. Rotating residents from outside hospitals were introduced to the HIV screening project by chief residents during their orientation.

PDSA cycle 8: This cycle saw the reintroduction of the HIV morning report presented by the chief medical resident to explain to the rotating residents on service the project and expectation. Forms with a general internal medicine history and physical template, with inclusion of HIV screening history and discussion included in the social history section was provided in the Internal Medicine resident workroom.

PDSA cycle 9: Continuation of the HIV morning report presented by the chief medical resident to explain to the rotating residents on service the project and expectation. Interns were instructed to provide facts regarding HIV infection, testing, or epidemiology during daily morning report meetings. Also, a competition between the twi wards teams for the highest proportion of HIV contemplation

PDSA cycle 10: No active intervention was taken regarding morning reports or chief resident reminders. However, a competition was initiated between the two teaching services. The service with a lower percent screened would provide the other service with an end of the month pizza party.

PDSA cycle 11: After the success of the team success of PDSA cycle 10 , the QI team intentionally decided to avoid active interventions during cycle 11 . There were no active interventions during the last month of the project including no morning report, no chief resident reminders and no resident provided HIV facts. There was also no competition between the two teaching services.

See supplementary file: ds7551.pdf - "Figure 2"

\section{Results}

Monthly rates of HIV screening contemplation were tabulated and compared (number of patients with HIV contemplation divided by number of patients in appropriate age range admitted to the IMResHospSvc). Since each PDSA cycle involved a different group of residents and attending physicians, we expected to observe variation in compliance.

The preintervention period was comprised of the 11 months of retrospective data collected before the intervention was initiated. The postintervention period refers to the 11 months of prospective data collected during the quality improvement project. Differences in proportions between the postintervention and preintervention periods were compared by using the chi-squared test. Data were analyzed using SAS 9.3 statistical software (SAS Institute Inc). All tests were two-sided, and statistical significance was defined as $\mathrm{P}<.05$.

The IMResHospSvc admitted 559 patients in the HIV-screening age range during the preintervention period. During the postintervention period, the IMResHospSvc admitted 1350 patients, of whom 541 $(40 \%)$ were in the HIV screening age group, with the remainder older than 64 years.

The number and percentage of documented preintervention and postintervention 1) offers to patients of HIV screening, 2) HIV tests ordered, and 3) physician HIV screening contemplation are summarized in table 3. Rates of all three improved. Specifically, postintervention offers of HIV screening increased significantly (7.9\% (44/559) vs. 55.5\% (300/541); $\mathrm{P}<.001)$, as did documentation of residents' contemplation of such screening (8.9\% (50/559) vs. $67.5 \%$ (365/541); $\mathrm{P}<.001)$. A significantly higher percentage of HIV screening tests was ordered postintervention (7.7\% (43/559) vs. $44.4 \%$ (240/541); $\mathrm{P}<.001)$. Monthly HIV screening documentation ranged from $0 \%(0 / 53)$ to $17.0 \%(9 / 53)$ preintervention, whereas it ranged from $30.6 \%(11 / 36)$ to $100 \%(62 / 62)$ postintervention.

The monthly percentage of patients offered HIV screening and the monthly percentage of patients whose physicians contemplated HIV screening are demonstrated in figure 2. There was improved but not sustained documentation of HIV contemplation in the postintervention year. The highest month of contemplation correlated with a PDSA cycle that utilized an active intervention of a 
non-cash prize competition between the teams. No new cases of HIV were identified during the course of the project.

See supplementary file: ds5964.docx - "Table 3 Statistics"

\section{Lessons and limitations}

We revised the interventions over time by compliance rates and our perceptions of intervention successes and barriers. We believe that transition of the educational curriculum presentation to the chief medical resident increased participation due to the superior administration position of the chief resident.

The friendly competition between the teams of the IMResHospSvc proved beneficial. A competition with a non-cash prize for the highest documented HIV contemplation at the end of the month prompted increased compliance, as it was the only PDSA cycle to result in $100 \%$ HIV contemplation. However, the benefit provided by team competition dissipated upon its removal, as demonstrated by decreased compliance $(58.8 \%(20 / 34))$.

Some barriers that presented variability were inherent to the system and could not be altered, such as the continual changes in IMResHospSvc residents and the older ages of inpatients. The QI interventions, as changed in response to the challenges posed by the results of the prior month, may have still been insufficient to accommodate the changing personalities and styles of each new set of residents. Some physician participants also indicated that establishing a habit of HIV screening was difficult because most (approximately 60\%) IMResHospSvc patients did not meet the age criterion, and thus a majority of patients admitted to the service were not appropriate for screening. The age criteria for HIV screening would not be a possible limitation in other health care systems where universal HIV screening is not limited to a certain age range. For example, the British HIV association does not specify age range for offering routine screening to individuals from a community with at least $1 \%$ HIV prevalence.[16]

We faced several barriers while conducting this QI project. Perhaps most unanticipated was the negative response from a few residents and attending physicians who expressed reluctance to participate. They indicated that the inpatient setting was inappropriate for HIV screening, despite the CDC recommendation to screen in all health care settings. Additionally, and interestingly, a few interns resisted the QI project, reportedly because they disliked being studied by colleagues. Since residents rotated onto and off the internal medicine service each month, this dissent possibly had limited impact.

Other factors affecting monthly variability in contemplation rates should be addressed in future interventions, including behavioral modification within the interventions, project fatigue, and indifference on the part of supervising physicians. We observed that active intervention measures were more effective but were also more time consuming without sustained improved compliance. This observation suggests that physician behavior is not permanently altered by active interventions. This finding has been demonstrated in other studies of behavioral modification, leading to the conclusion that an effective and universal behavior modification has yet to be developed.[10, 11] Previous studies in professional practices have demonstrated that, when initial compliance is low, active interventions can produce a modest increase in overall compliance to a given standard.[11]

Finally, there was no incentive to supervising attending physicians to encourage or require resident adherence to the CDC guidelines or the goals of this $\mathrm{Ql}$ project, nor were there any repercussions if they chose not to do so. Their apparent indifference may have limited the incentive for residents and interns to incorporate HIV screening into patient evaluations, since it was not routinely requested by the supervising attending physician.

Despite these barriers and unforeseen variables, our interventions did produce a modest improvement in overall compliance with the CDC guidelines for HIV screening. The long term effects of the interventions within our system have yet to be weighed. A future study aimed at improving adherence to HIV screening guidelines might evaluate the benefits of point of care prompting. Such prompting has been studied primarily with the introduction of the $E H R$, and several authors have found that it increases compliance with documentation and intervention guidelines.[12-14] Computerized prompting has improved even moderate compliance with vaccination, deep vein thrombosis prophylaxis, and aspirin use.[12] Computerized prompting has also played a role in antibiotic use compliance, with the most profound improvement in the perioperative setting, where compliance improved from $40 \%$ before point-of-care prompting to $99.1 \%$ after its introduction.[13] Although point-of-care prompting has not always improved compliance,[15] it may do so when used in addition to the types of interventions used in our QI project. Electronic prompting might also potentiate a more durable effect.

\section{Conclusion}

Our QI project produced modest improvement in compliance with the CDC's HIV screening guidelines through combined resident education, individualized reminders, and team competition. This intervention could be implemented in another residency program or hospital service, since it demonstrated improvement in guideline adherence. However, our findings indicate that behavioral modification is labor intensive, with variable results even in a highly educated population. Further study is required to assess the durability of these interventions. Additional improvement in compliance and durable effects may be generated by point of care prompting by the EHR.

\section{References}

1. Accreditation Council for Graduate Medical Education (ACGME). Common Program Requirement [Internet]. 2009 Jan 15 [cited 2014 May 15]; Available from: http://www.acgme.org/acgmeweb/Portals/0/PDFs/commong uide/IVA5c_EducationalProgram_ACGMECompetencies_P BLI Explanation.pdf.

2. US Preventive Services Task Force. Screening for HIV 
[Internet]. 2013 Apr [cited 2014 May 15]. Available from: http://www.uspreventiveservicestaskforce.org/uspstf/uspshiv i.htm.

3. Alpert PL, Shuter J, DeShaw MG, Webber MP, Klein RS. Factors associated with unrecognized HIV-1 infection in an inner-city emergency department. Ann Emerg Med

1996 Aug;28(2):159-64.

1. Liddicoat RV, Horton NJ, Urban R, Maier E, Christiansen D, Samet JH. Assessing missed opportunities for HIV testing in medical settings. J Gen Intern Med 2004 Apr;19(4):349-56.

2. Ruiz MS, Gable AR, Kaplan EH, Stoto MA, Fineberg HV, Trussell J, editors. No time to lose: getting more from HIV prevention. Division of Health Promotion and Disease Prevention, Washington, DC: National Academies Press; 2001.

3. Branson BM, Handsfield HH, Lampe MA, Janssen RS, Taylor AW, Lyss SB, et al.; Centers for Disease Control and Prevention (CDC). Revised recommendations for HIV testing of adults, adolescents, and pregnant women in health-care settings. MMWR Recomm Rep 2006 Sep 22;55(RR-14):1-17.

4. Arizona Department of Health Services. HIV epidemiology program: annual report-2013. [Internet] [Updated 2014 Mar 17; cited 2014 May 15]. Available from: http://www.azdhs.gov/phs/edc/odis/hivepidemiology/reports/index.php.

5. Arizona Department of Health Services. Surveillance data supplemental material. [Internet] [Updated 2014 Mar 17; cited 2014 May 15]. Available from:

http://www.azdhs.gov/phs/edc/odis/documents/hiv-epidemiol ogy/2013/hiv-surveillance-data-supplemental-material.pdf.

6. Arizona Department of Health Services, Bureau of Health Systems Development. Scottsdale Special Area (SArea): Statistical profile-2012. [Internet] 2013 Apr 23 [cited 2014 May 29]. Available from:

http://www.azdhs.gov/hsd/data/profiles/documents/townscities/maricopa/65000.pdf/.

7. Abraham C, Michie S. A taxonomy of behavior change techniques used in interventions. Health Psychol 2008 May;27(3):379-87.

8. Ivers N, Jamtvedt G, Flottorp S, Young JM, OdgaardJensen J, French SD, et al. Audit and feedback: effects on professional practice and healthcare outcomes. Cochrane Database Syst Rev 2012 Jun 13;6:CD000259.

9. Dexter PR, Perkins S, Overhage JM, Maharry K, Kohler RB, McDonald CJ. A computerized reminder system to increase the use of preventive care for hospitalized patients. N Engl J Med 2001 Sep 27;345(13):965-70.

10. Pestotnik SL, Classen DC, Evans RS, Burke JP. Implementing antibiotic practice guidelines through computer-assisted decision support: clinical and financial outcomes. Ann Intern Med 1996 May 15;124(10):884-90.

11. Haberman S, Feldman J, Merhi ZO, Markenson G, Cohen $\mathrm{W}$, Minkoff $\mathrm{H}$. Effect of clinical-decision support on documentation compliance in an electronic medical record. Obstet Gynecol. 2009 Aug;114(2 Pt 1):311-7.
12. Murray MD, Harris LE, Overhage JM, Zhou XH, Eckert GJ, Smith FE, et al. Failure of computerized treatment suggestions to improve health outcomes of outpatients with uncomplicated hypertension: results of a randomized controlled trial. Pharmacotherapy 2004;24(3):324-37.

13. British HIV Association (BHIVA). UK National Guidelines for HIV Testing 2008. September 2008. Available at See www.bhiva.orgHIVTesting2008.aspx (last accessed 2 April 2016).

\section{Declaration of interests}

Nothing to declare.

\section{Acknowledgements}

We would like to acknowledge the Mayo Arizona Internal Medicine Department for their support of the internal medicine residency program and our research ventures.

\section{Ethical approval}

According to the policy activities that constitute research at Mayo Clinic Arizona this work met criteria for operational improvement activities exempt from ethics review. 